\title{
Cost Benefit Analysis of Series Systems with Mixed Standby Components and $k$-stage Erlang Repair Time
}

\author{
Mohamed Salah EL-Sherbeny
}

Department of Mathematics, Faculty of Science, Helwan University Cairo, P. O. Box 11795, Egypt

\begin{abstract}
In this paper, we compare the availability characteristics between three different series system configurations with mixed (cold and warm) standby components and $k$-stage Erlang repair times. Three configurations are studied under the assumption that the time-to-failure for each of the operative and warm standby components are assumed to be exponentially distributed with parameters $\lambda$ and $\alpha$, respectively. We present a recursive method, using the supplementary variable technique; we develop the explicit expressions for the steady-state availability. Under the cost/benefit criterion, comparisons are made based on assumed numerical values given to the distribution parameters, and to the cost of the operative and standby units.
\end{abstract}

Keywords Availability, Cost-Benefit, Erlang Distribution, Supplementary Variable, Series System

\section{Introduction}

This type of problems is discussed by $[2,3]$ have studied a two similar or dissimilar unit cold standby redundant system with preventive maintenance, inspection and two types of repairs.[4] carried out the cost-benefit analysis of a one-server two-unit system subject to different repair policies. They carried out the analysis under the assumption that a unit undergoes only one type of failure with no specific mention about inspection. [5, 6] have studied the stochastic behaviour of some standby redundant systems.[9] considered the reliability and mean time to system failure (MTTF) analysis of a two-state complex with repairable system, consisting of two sub-systems A and B arranged in series, incorporating the concept of hardware and human failures.[7] considered reliability, availability and cost/benefit of four different series system configurations with mixed standby components.[11] studied a model representing a two-unit active and one-unit on standby human-machine system with general failed system repair time distribution. A two-unit warm standby system with constant failure rate and two types of repairmen and patience time were investigated by[8].[1] studied the two unit standby system and obtained exact confidence limits for the steady-state availability of the system, when the failure rate of an operative unit is constant and the repair time of the failed unit is a two stage Erlang distribution.[10] studied the optimal system for series systems with mixed standby components.

\section{* Corresponding author:}

m_el_sherbeny@yahoo.com (Mohamed Salah EL-Sherbeny)

Published online at http://journal.sapub.org/ijps

Copyright (C) 2012 Scientific \& Academic Publishing. All Rights Reserved
The main contribution of this paper is three folds. First, we present a recursive method, using the supplementary variable technique and treating the supplementary variable as the remaining repair time, to develop the $A v_{i}(\infty)$, for configuration $i$, where $i=1,2,3$. The second purpose is to develop the explicit expressions for the steady-state availability, for the three configurations. Finally, we rank three configurations for the $A v_{i}(\infty)$ based on assumed numerical values given to the system parameters.

\section{Assumptions and Configurations Description}

We consider a power plant of $10 \mathrm{MW}$ satisfies the following assumptions:

1. The system comprises of operative components and mixed standby components "cold and warm".

2. The generators are available in components of both 10 and $5 \mathrm{MW}$.

3. Standby generators are always necessary in case of failure.

4. When the operative component fails, it is immediately replaced by a warm standby if it is available and the cold standby becomes warm standby component.

5 . We assume that the switch is perfect and the switchover time from warm standby component to primary component, from cold standby component to warm standby component, from failure to repair, or from repair to cold standby component (or primary component if the system is short) is instantaneous.

6. When operative and warm standby components are repaired, they become as good as new. 
7. The operative and warm standby components fail independently of the others and follow an exponential time to failure distribution with parameters $\lambda$ and $\alpha$ respectively.

8. The time to repair of the components is independent and identically distributed random variable following $\mathrm{k}$-stage Erlang distribution with probability density function $b(u)$ and mean repair time $\frac{1}{\mu}$.

where

$$
b(u)=\frac{(\mu k)^{k}}{(k-1) !} u^{k-1} e^{-\mu k u}, 0<u<\infty, \mu>0 .
$$

9. If one operative unit or warm standby unit is in repair, then arriving failed units have to wait in the queue until the server is available.

The above assumptions are common to all of the following three configurations:

Configuration 1 is a serial system of one operative $10 \mathrm{MW}$ component, one warm standby $10 \mathrm{MW}$ component and one cold 10 MW component.

Configuration 2 is a serial system of two operative $5 \mathrm{MW}$ components, one warm standby $5 \mathrm{MW}$ component and one cold $5 \mathrm{MW}$ component.

Configuration 3 is a serial system of one operative $10 \mathrm{MW}$ component, two warm standby $10 \mathrm{MW}$ components and one cold 10 MW component.

\section{Cost-benefit factor}

In this paper, we assume that the size-proportional costs for the operative components and warm standby components are given in Table 1. With this, we calculate the costs for each configuration $i(i=1,2,3)$ which are shown in Table 2 . Throughout this paper, we assume that $C_{i}$ be the cost of configuration $i$ and $F_{i}$ the benefit of configuration $i$, where $F_{i}$ is $A v_{i}(\infty)$.

Table 1. The size-proportional cost for the operative, warm and cold standby components

\begin{tabular}{|cc|}
\hline Component & Cost(in \$) \\
Operative 10MW & $10 \times 10^{6}$ \\
Operative 5MW & $5 \times 10^{6}$ \\
Warm standby 10MW & $6 \times 10^{6}$ \\
Warm standby 5MW & $3 \times 10^{6}$ \\
Cold standby 10MW & $4 \times 10^{6}$ \\
Cold standby 5MW & $2 \times 10^{6}$ \\
\hline
\end{tabular}

Table 2. The costs for each configuration $i(i=1,2,3)$

\begin{tabular}{|cc|}
\hline Configuration & Cost(in \$) \\
Configuration 1 & $20 \times 10^{6}$ \\
Configuration 2 & $15 \times 10^{6}$ \\
Configuration 3 & $24 \times 10^{6}$ \\
\hline
\end{tabular}

\section{Availability Analysis of the System}

We use the following supplementary variable: $U \equiv$ remaining repair time for the component under repair. The state of the system at time $t$ is given by

$N(t) \equiv$ number of working units in the system; and
$U(t) \equiv$ remaining repair time for the component being repaired.

and define

$$
P_{n}(u, t) d u=P\{N(t)=n, u<U(t) \leq u+d u\}, \quad u \geq 0,
$$

and $P_{n}(t)=\int_{0}^{\infty} P_{n}(u, t) d$

\subsection{Availability for Configuration 1}

Relating the state of the system at time $t$ and $t+d t$, we obtain:

$$
\frac{d}{d t} P_{3}(t)=-(\lambda+\alpha) P_{3}(t)+P_{2}(0, t),
$$

$$
\begin{gathered}
\left(\frac{\partial}{\partial t}-\frac{\partial}{\partial u}\right) P_{2}(u, t)=-(\lambda+\alpha) P_{2}(u, t)+(\lambda+\alpha) P_{3}(u, t)+b(u) P_{1}(0, t) \\
\left(\frac{\partial}{\partial t}-\frac{\partial}{\partial u}\right) P_{1}(u, t)=-\lambda P_{1}(u, t)+(\lambda+\alpha) P_{2}(u, t)+b(u) P_{0}(0, t) \\
\left(\frac{\partial}{\partial t}-\frac{\partial}{\partial u}\right) P_{0}(u, t)=\lambda P_{1}(u, t)
\end{gathered}
$$

In steady-state, let us define

$$
\begin{gathered}
P_{n}=\lim _{t \rightarrow \infty} P_{n}(t), n=3,2,1,0 . \\
P_{n}(u)=\lim _{t \rightarrow \infty} P_{n}(u, t), n=3,2,1,0 .
\end{gathered}
$$

and further define

$$
P_{3}(u)=b(u) P_{3},
$$

From (1)-(5), we obtain the following steady-state equations as follow:

$$
\begin{gathered}
0=-(\lambda+\alpha) P_{3}+P_{2}(0), \\
-\frac{d}{d u} P_{2}(u)=-(\lambda+\alpha) P_{2}(u)+(\lambda+\alpha) b(u) P_{3}+b(u) P_{1}(0), \\
-\frac{d}{d u} P_{1}(u)=-\lambda P_{1}(u)+(\lambda+\alpha) P_{2}(u)+b(u) P_{0}(0), \\
-\frac{d}{d u} P_{0}(u)=\lambda P_{1}(u) .
\end{gathered}
$$

Now, from (6), we obtain:

$$
P_{2}(0)=(\lambda+\alpha) P_{3},
$$

Further, we define

$$
\begin{gathered}
B^{*}(s)=\int_{0}^{\infty} e^{-s u} b(u) d u=\left(\frac{k \mu}{k \mu+s}\right)^{k}, \\
P_{n}^{*}(s)=\int_{0}^{\infty} e^{-s u} P_{n}(u) d u, \quad P_{n}=P_{n}^{*}(0)=\int_{0}^{\infty} P_{n}(u) d u,
\end{gathered}
$$

and $\int_{0}^{\infty} e^{-s u} \frac{d}{d u} P_{n}(u) d u=s P_{n}^{*}(s)-P_{n}(0)$.

Now, taking the Laplace- Stieltjes Transform (LST) on both sides of (7)-(9) and using (10) yield:

$$
\begin{gathered}
(\lambda+\alpha-s) P_{2}^{*}(s)=\left(B^{*}(s)-1\right) P_{2}(0)+B^{*}(s) P_{1}(0) \\
(\lambda-s) P_{1}^{*}(s)=(\lambda+\alpha) P_{2}^{*}(s)+B^{*}(s) P_{0}(0)-P_{1}(0) \\
s P_{0}^{*}(s)=P_{0}(0)-\lambda P_{1}^{*}(s)
\end{gathered}
$$

We develop a recursive method to get the explicit ex- 
pressions $P_{n}^{*}(0)(n=2,1,0)$.

Setting $s=\lambda+\alpha$ and $s=0$ in (11), respectively yields

$$
P_{1}(0)=\frac{1-B^{*}(\lambda+\alpha)}{B^{*}(\lambda+\alpha)} P_{2}(0)=\frac{(\lambda+\alpha)\left(1-B^{*}(\lambda+\alpha)\right)}{B^{*}(\lambda+\alpha)} P_{3},
$$

and,

$$
P_{2}^{*}(0)=\frac{1}{(\lambda+\alpha)} P_{1}(0)=\frac{\left(1-B^{*}(\lambda+\alpha)\right)}{B^{*}(\lambda+\alpha)} P_{3} .
$$

Again, setting $s=\lambda$ in (11), it follows that:

$$
P_{2}^{*}(\lambda)=\frac{(\lambda+\alpha)\left(B^{*}(\lambda)-B^{*}(\lambda+\alpha)\right)}{\alpha B^{*}(\lambda+\alpha)} P_{3} .
$$

Setting $s=\lambda$ in (12) yields:

$$
P_{0}(0)=\frac{P_{1}(0)-(\lambda+\alpha) P_{2}^{*}(\lambda)}{B^{*}(\lambda)} .
$$

Substituting (14) and (16) in (17), we have:

$$
P_{0}(0)=\left\{\frac{(\lambda+\alpha)\left[\alpha\left(1-B^{*}(\lambda+\alpha)\right)-(\lambda+\alpha)\left(B^{*}(\lambda)-B^{*}(\lambda+\alpha)\right)\right]}{\alpha B^{*}(\lambda) B^{*}(\lambda+\alpha)}\right\} P_{3} .
$$

Similarly, setting $s=0$ in equation (12), we obtain:

$$
\lambda P_{1}^{*}(0)=(\lambda+\alpha) P_{2}^{*}(0)+P_{0}(0)-P_{1}(0) .
$$

From (19) and (15), we have:

$$
P_{1}^{*}(0)=\frac{1}{\lambda} P_{0}(0) \text {. }
$$

Thus

$P_{1}^{*}(0)=\frac{(\lambda+\alpha)}{\lambda}\left\{\frac{\left[\alpha\left(1-B^{*}(\lambda+\alpha)\right)\right]-(\lambda+\alpha)\left(B^{*}(\lambda)-B^{*}(\lambda+\alpha)\right)}{\alpha B^{*}(\lambda) B^{*}(\lambda+\alpha)}\right\} P_{3}$.

Differentiating (13) with respect to $\mathrm{s}$ and setting $\mathrm{s}=0$ in the result, we obtain:

$$
P_{0}^{*}(0)=-\lambda P_{1}^{*(1)}(0) .
$$

Differentiating (11) with respect to $\mathrm{s}$ and then setting $\mathrm{s}=0$ in the result we have:

$$
(\lambda+\alpha) P_{2}^{*(1)}(0)=P_{2}^{*}(0)-\frac{\left[P_{2}(0)+P_{1}(0)\right]}{\mu} .
$$

Similarly, differentiating (12) with respect to s and setting $\mathrm{s}=0$ in the result, we find that:

$$
\lambda P_{1}^{*(1)}(0)=P_{1}^{*}(0)+P_{2}^{*}(0)-\frac{\left[P_{2}(0)+P_{1}(0)+P_{0}(0)\right]}{\mu} .
$$

Substituting (23) in (21), we have:

$$
P_{0}^{*}(0)=\frac{\left[P_{2}(0)+P_{1}(0)+P_{0}(0)\right]}{\mu}-\left(P_{2}^{*}(0)+P_{1}^{*}(0)\right) .
$$

where $P_{2}^{*}(0), P_{2}(0), P_{1}(0)$ and $P_{0}(0)$ are given in (15), (10), (14) and (18), respectively.

Now, using the normalizing condition

$$
P_{3}+P_{2}^{*}(0)+P_{1}^{*}(0)+P_{0}^{*}(0)=1,
$$

We can compute $P_{3}$ as follow:

$$
P_{3}=\frac{\alpha \mu B^{*}(\lambda) B^{*}(\lambda+\alpha)}{\left[(\lambda+\alpha)\left(\alpha-\lambda B^{*}(\lambda)\right)+\left(\lambda(\lambda+\alpha)+\alpha \mu B^{*}(\lambda)\right) B^{*}(\lambda+\alpha)\right]} .
$$

The explicit expression for the availability of configuration $1\left(A v_{1}(\infty)\right)$ is given by:

$$
A v_{1}(\infty)=P_{3}+P_{2}^{*}(0)+P_{1}^{*}(0) .
$$

Using (15), (20), (25) and (26), we obtain the explicit expression for the $A v_{1}(\infty)$

$$
A v_{1}(\infty)=\frac{\gamma_{1}}{\varpi_{1}} .
$$

where

$$
\gamma_{1}=\mu\left[-\left(\alpha^{2}+\alpha \lambda+\lambda^{2}\right) B^{*}(\lambda)+(\alpha+\lambda)\left(\alpha+\lambda B^{*}(\lambda+\alpha)\right)\right],
$$

and

$$
\varpi_{1}=\lambda(\alpha+\lambda)\left(\alpha+\lambda B^{*}(\lambda+\alpha)\right)-\lambda B^{*}(\lambda)\left(\lambda(\lambda+\alpha)-\alpha \mu B^{*}(\lambda+\alpha)\right) .
$$

\subsection{Availability for Configuration 2}

Following the same procedures in the subsection 3.1, we can get the steady-state equations as follow:

$$
0=-(2 \lambda+\alpha) P_{4}+P_{3}(0),
$$

$$
\begin{gathered}
-\frac{d}{d u} P_{3}(u)=-(2 \lambda+\alpha) P_{3}(u)+(2 \lambda+\alpha) b(u) P_{4}+b(u) P_{2}(0), \\
-\frac{d}{d u} P_{2}(u)=-2 \lambda P_{2}(u)+(2 \lambda+\alpha) P_{3}(u)+b(u) P_{1}(t) \\
-\frac{d}{d u} P_{1}(u)=2 \lambda P_{2}(u) .
\end{gathered}
$$

Now, from (28), we obtain:

$$
P_{3}(0)=(2 \lambda+\alpha) P_{4},
$$

Taking the LST on both sides of (29)-(31) and using (32), we get that:

$$
\begin{gathered}
(2 \lambda+\alpha-s) P_{3}^{*}(s)=\left(B^{*}(s)-1\right) P_{3}(0)+B^{*}(s) P_{2}(0), \\
(2 \lambda-s) P_{2}^{*}(s)=(2 \lambda+\alpha) P_{3}^{*}(s)+B^{*}(s) P_{1}(0)-P_{2}(0), \\
s P_{1}^{*}(s)=P_{1}(0)-2 \lambda P_{2}^{*}(s)
\end{gathered}
$$

Setting $s=2 \lambda+\alpha$ and $s=0$ in (33), respectively yields:

$P_{2}(0)=\frac{1-B^{*}(2 \lambda+\alpha)}{B^{*}(2 \lambda+\alpha)} P_{3}(0)=\frac{(2 \lambda+\alpha)\left(1-B^{*}(2 \lambda+\alpha)\right)}{B^{*}(2 \lambda+\alpha)} P_{4}$,

and

$$
P_{3}^{*}(0)=\frac{1}{(2 \lambda+\alpha)} P_{2}(0)=\frac{\left(1-B^{*}(2 \lambda+\alpha)\right)}{B^{*}(2 \lambda+\alpha)} P_{4} .
$$

Again, setting $s=2 \lambda$ in (33), it follows that:

$$
P_{3}^{*}(2 \lambda)=\frac{\left(B^{*}(2 \lambda)-1\right) P_{3}(0)+B^{*}(2 \lambda) P_{2}(0)}{\alpha}
$$

Setting $s=2 \lambda$ in (34) yields:

$$
P_{1}(0)=\frac{P_{2}(0)-(2 \lambda+\alpha) P_{3}^{*}(2 \lambda)}{B^{*}(2 \lambda)} .
$$

Substituting (36) and (38) in (39), we have:

$P_{1}(0)=(2 \lambda+\alpha)\left\{\frac{\left[\alpha\left(1-B^{*}(2 \lambda+\alpha)\right)\right]-(2 \lambda+\alpha)\left(B^{*}(2 \lambda)-B^{*}(2 \lambda+\alpha)\right)}{\alpha B^{*}(2 \lambda) B^{*}(2 \lambda+\alpha)}\right\} P_{4}$

Similarly, setting $s=0$ in (34), we obtain:

$$
2 \lambda P_{2}^{*}(0)=(2 \lambda+\alpha) P_{3}^{*}(0)+P_{1}(0)-P_{2}(0) .
$$

From above equation and (37), we have:

$$
P_{2}^{*}(0)=\frac{(2 \lambda+\alpha) P_{3}^{*}(0)+P_{1}(0)-P_{2}(0)}{2 \lambda} .
$$


Now, using (40), we get:

$P_{2}^{*}(0)=\frac{(2 \lambda+\alpha)}{2 \lambda}\left\{\frac{\left[\alpha\left(1-B^{*}(2 \lambda+\alpha)\right)\right]-(2 \lambda+\alpha)\left(B^{*}(2 \lambda)-B^{*}(2 \lambda+\alpha)\right)}{\alpha B^{*}(2 \lambda) B^{*}(2 \lambda+\alpha)}\right\} P_{4}$

Differentiating (35) with respect to $\mathrm{s}$ and setting $\mathrm{s}=0$ in the result, we obtain:

$$
P_{1}^{*}(0)=-2 \lambda P_{2}^{*(1)}(0) \text {. }
$$

Differentiating (33) with respect to $\mathrm{s}$ and then setting $\mathrm{s}=0$ in the result yields:

$$
(2 \lambda+\alpha) P_{3}^{*(1)}(0)=P_{3}^{*}(0)-\frac{\left[P_{3}(0)+P_{2}(0)\right]}{\mu} .
$$

Likewise, differentiating (34) with respect to s and setting $\mathrm{s}=0$ in the result, we find that:

$$
2 \lambda P_{2}^{*(1)}(0)=P_{2}^{*}(0)+P_{3}^{*}(0)-\frac{\left[P_{3}(0)+P_{2}(0)+P_{1}(0)\right]}{\mu} .
$$

Substituting (44) in (42), we have:

$$
P_{1}^{*}(0)=\frac{\left[P_{3}(0)+P_{2}(0)+P_{1}(0)\right]}{\mu}-\left(P_{2}^{*}(0)+P_{3}^{*}(0)\right),
$$

where $P_{3}^{*}(0), P_{3}(0), P_{2}(0)$ and $P_{1}(0)$ are given in (37),(32),(36) and (40), respectively.

Now, using the normalizing condition:

$$
P_{4}+P_{3}^{*}(0)+P_{2}^{*}(0)+P_{1}^{*}(0)=1,
$$

We can compute $P_{4}$ as follow.

$$
P_{4}=\frac{\alpha \mu B^{*}(2 \lambda) B^{*}(2 \lambda+\alpha)}{\left[\begin{array}{l}
(2 \lambda+\alpha)\left(\alpha-2 \lambda B^{*}(2 \lambda)\right) \\
+\left(2 \lambda(2 \lambda+\alpha)+\alpha \mu B^{*}(2 \lambda)\right) B^{*}(2 \lambda+\alpha)
\end{array}\right]} .
$$

The availability of configuration $2\left(A v_{2}(\infty)\right)$ is given by:

$$
A v_{2}(\infty)=P_{4}+P_{3}^{*}(0)+P_{2}^{*}(0) \text {. }
$$

Subsisting (37), (41) and (47) into (48), we obtain the $A v_{2}(\infty)$ :

$$
A v_{2}(\infty)=\frac{\gamma_{2}}{\varpi_{2}}
$$

where

$$
\gamma_{2}=\mu\left[-\left(\alpha^{2}+2 \alpha \lambda+4 \lambda^{2}\right) B^{*}(2 \lambda)+(\alpha+2 \lambda)\left(\alpha+2 \lambda B^{*}(2 \lambda+\alpha)\right)\right],
$$

and

$$
\varpi_{2}=2 \lambda\left[(\alpha+2 \lambda)\left(\alpha-2 \lambda B^{*}(2 \lambda)\right)+\left(2 \lambda(2 \lambda+\alpha)+\alpha \mu B^{*}(2 \lambda)\right) B^{*}(2 \lambda+\alpha)\right] .
$$

\subsection{Availability for Configuration 3}

We use the same procedure as above to obtain the steady-state equations as follow:

$$
\begin{gathered}
0=-(\lambda+2 \alpha) P_{4}+P_{3}(0), \\
-\frac{d}{d u} P_{3}(u)=-(\lambda+2 \alpha) P_{3}(u)+(\lambda+2 \alpha) b(u) P_{4}+b(u) P_{2}(0), \\
-\frac{d}{d u} P_{2}(u)=-(\lambda+\alpha) P_{2}(u)+(\lambda+2 \alpha) P_{3}(u)+b(u) P_{1}(0), \\
-\frac{d}{d u} P_{1}(u)=-\lambda P_{1}(u)+(\lambda+\alpha) P_{2}(u)+b(u) P_{0}(0),
\end{gathered}
$$

$$
-\frac{d}{d u} P_{0}(u)=\lambda P_{1}(u)
$$

Now, from (50), we obtain:

$$
P_{3}(0)=(\lambda+2 \alpha) P_{4} .
$$

Taking the LST on both sides of (51)-(54) and using (55), we obtain:

$$
\begin{gathered}
(\lambda+2 \alpha-s) P_{3}^{*}(s)=\left(B^{*}(s)-1\right) P_{3}(0)+B^{*}(s) P_{2}(0) \\
(\lambda+\alpha-s) P_{2}^{*}(s)=(\lambda+2 \alpha) P_{3}^{*}(s)+B^{*}(s) P_{1}(0)-P_{2}(0) \\
(\lambda-s) P_{1}^{*}(s)=(\lambda+\alpha) P_{2}^{*}(s)+B^{*}(s) P_{0}(0)-P_{1}(0) \\
s P_{0}^{*}(s)=P_{0}(0)-\lambda P_{1}^{*}(s)
\end{gathered}
$$

Setting $s=\lambda+2 \alpha, s=0, s=\lambda+\alpha$ and $s=\lambda$ in (56), respectively yields:

$$
\begin{gathered}
P_{2}(0)=\frac{1-B^{*}(\lambda+2 \alpha)}{B^{*}(\lambda+2 \alpha)} P_{3}(0)=\frac{(\lambda+2 \alpha)\left(1-B^{*}(\lambda+2 \alpha)\right)}{B^{*}(\lambda+2 \alpha)} P_{4}, \\
P_{3}^{*}(0)=\frac{P_{2}(0)}{(\lambda+2 \alpha)}=\frac{\left(1-B^{*}(\lambda+2 \alpha)\right)}{B^{*}(\lambda+2 \alpha)} P_{4}, \\
P_{3}^{*}(\lambda+\alpha)=\frac{(\lambda+2 \alpha)\left(B^{*}(\lambda+\alpha)-B^{*}(\lambda+2 \alpha)\right)}{\alpha B^{*}(\lambda+2 \alpha)} P_{4}, \\
P_{3}^{*}(\lambda)=\frac{\left(B^{*}(\lambda)-1\right) P_{3}(0)+B^{*}(\lambda) P_{2}(0)}{2 \alpha} .
\end{gathered}
$$

Again, setting $s=\lambda+\alpha, s=0$ and $s=\lambda$ in (57), respectively yields:

$$
\begin{gathered}
P_{1}(0)=\frac{P_{2}(0)-(\lambda+2 \alpha) P_{3}^{*}(\lambda+\alpha)}{B^{*}(\lambda+\alpha)}, \\
P_{2}^{*}(0)=\frac{(\lambda+2 \alpha) P_{3}^{*}(0)+P_{1}(0)-P_{2}(0)}{(\lambda+\alpha)}, \\
P_{2}^{*}(\lambda)=\frac{(\lambda+2 \alpha) P_{3}^{*}(\lambda)+B^{*}(\lambda) P_{1}(0)-P_{2}(0)}{\alpha} .
\end{gathered}
$$

Likewise, setting $s=\lambda$ and $s=0$ in (58), we obtain:

$$
\begin{aligned}
P_{0}(0) & =\frac{P_{1}(0)-(\lambda+\alpha) P_{2}^{*}(\lambda)}{B^{*}(\lambda)}, \\
P_{1}^{*}(0) & =\frac{(\lambda+\alpha) P_{2}^{*}(0)+P_{0}(0)-P_{1}(0)}{\lambda} .
\end{aligned}
$$

Differentiating (59) with respect to $\mathrm{s}$ and setting $\mathrm{s}=0$ in the result, we obtain:

$$
P_{0}^{*}(0)=-\lambda P_{1}^{*(1)}(0) .
$$

Differentiating (56) with respect to $\mathrm{s}$ and then setting $\mathrm{s}=0$ in the result yields:

$$
(\lambda+2 \alpha) P_{3}^{*(1)}(0)=P_{3}^{*}(0)-\frac{\left[P_{3}(0)+P_{2}(0)\right]}{\mu} .
$$

Differentiating (57) with respect to $\mathrm{s}$ and then setting $\mathrm{s}=0$ in the result, it follows that:

$$
(\lambda+\alpha) P_{2}^{*(1)}(0)=P_{2}^{*}(0)+P_{3}^{*}(0)-\frac{\left[P_{3}(0)+P_{2}(0)+P_{1}(0)\right]}{\mu} .
$$


Likewise, differentiating (58) with respect to s and setting $\mathrm{s}=0$ in the result, we find that:

$\lambda P_{1}^{*(1)}(0)=P_{1}^{*}(0)+P_{2}^{*}(0)+P_{3}^{*}(0)-\frac{\left[P_{3}(0)+P_{2}(0)+P_{1}(0)+P_{0}(0)\right]}{\mu}$.

Substituting (72) into (69), we have:

$P_{0}^{*}(0)=\frac{\left[P_{3}(0)+P_{2}(0)+P_{1}(0)+P_{0}(0)\right]}{\mu}-\left(P_{3}^{*}(0)+P_{1}^{*}(0)+P_{2}^{*}(0)\right)$

Now, using the normalizing condition:

$$
P_{4}+P_{3}^{*}(0)+P_{2}^{*}(0)+P_{1}^{*}(0)+P_{0}^{*}(0)=1,
$$

We can compute $P_{4}$ as follow:

$$
P_{4}=\frac{-2 \alpha^{2} \mu B^{*}(\lambda) B^{*}(\lambda+\alpha) B^{*}(\lambda+2 \alpha)}{\psi} .
$$

where

$$
\begin{aligned}
& \psi=\left[(\lambda+2 \alpha)\left(\begin{array}{l}
2 \alpha^{2}\left(-1+B^{*}(\alpha+\lambda)\right) \\
+\lambda B^{*}(\lambda)\left(2 \alpha-(\alpha+\lambda) B^{*}(\alpha+\lambda)\right)
\end{array}\right)-(2(\lambda+\alpha)(\lambda+2 \alpha)\right. \\
& \left.\left.\left(\alpha-\lambda B^{*}(\lambda)\right)+\left(\begin{array}{l}
\lambda(\lambda+\alpha)(\lambda+2 \alpha) \\
+2 \alpha^{2} \mu B^{*}(\lambda)
\end{array}\right) B^{*}(\lambda+\alpha)\right) B^{*}(\lambda+2 \alpha)\right] .
\end{aligned}
$$

The availability of configuration $3\left(A v_{3}(\infty)\right)$ is given by:

$$
A v_{3}(\infty)=P_{4}+P_{3}^{*}(0)+P_{2}^{*}(0)+P_{1}^{*}(0) .
$$

Using (61), (65), (68), (75) and (76), we obtain the $A v_{3}(\infty)$ :

$$
A v_{3}(\infty)=\frac{\gamma_{3}}{\varpi_{3}} .
$$

where

$$
\begin{gathered}
\gamma_{3}=\mu\left[( 2 \alpha + \lambda ) ( \alpha + \lambda ) \left(-2 \alpha^{2}\left(\begin{array}{l}
\left.-1+B^{*}(\alpha+\lambda)\right) \\
+(\alpha+\lambda)\left(2 \alpha+\lambda B^{*}(\alpha+\lambda)\right) B^{*}(2 \alpha+\lambda)
\end{array}\right)\right.\right. \\
+B^{*}(\lambda)\left(\left(\begin{array}{l}
4 \alpha^{4}+6 \alpha^{3} \lambda \\
+7 \alpha^{2} \lambda^{2}+4 \alpha \lambda^{3}+\lambda^{4}
\end{array}\right) B^{*}(\alpha+\lambda)-2(2 \alpha+\lambda)\left(\alpha^{2}+\alpha \lambda+\lambda^{2}\right)\right. \\
\left.\left.\left(\alpha+(\alpha+\lambda) B^{*}(2 \alpha+\lambda)\right)\right)\right]
\end{gathered}
$$

and

$$
\begin{aligned}
\varpi_{3}= & \lambda(\alpha+\lambda)\left[(2 \alpha+\lambda)\left(\begin{array}{l}
-2 \alpha^{2}\left(-1+B^{*}(\alpha+\lambda)\right) \\
+(\alpha+\lambda)\left(2 \alpha+\lambda B^{*}(\alpha+\lambda)\right) B^{*}(2 \alpha+\lambda)
\end{array}\right)\right. \\
& +B^{*}(\lambda)\left(-2 \lambda(2 \alpha+\lambda)\left(\alpha+(\alpha+\lambda) B^{*}(2 \alpha+\lambda)\right)\right. \\
& \left.\left.+B^{*}(\alpha+\lambda)\left(\lambda(\alpha+\lambda)(2 \alpha+\lambda)+2 \alpha^{2} \mu B^{*}(2 \alpha+\lambda)\right)\right)\right]
\end{aligned}
$$

\section{Comparison between the three Configurations}

The purpose of this section is to compare $A v_{i}(\infty)$ for $i=1,2,3$ when $\lambda=0.001, \mu=0.1, \alpha=0.2 \lambda$ and $k=1,2,3$.

Comparisons for the $A v_{i}(\infty)$

Three cases are illustrated in Tables 3-10.

\begin{tabular}{|c|c|c|c|}
\hline$\lambda$ & $A v_{1}(\infty)$ & $A v_{2}(\infty)$ & $A v_{3}(\infty)$ \\
\hline 0.001 & 0.999999 & 0.999995 & 1.000 \\
\hline 0.002 & 0.999995 & 0.999965 & 1.000 \\
\hline 0.003 & 0.999983 & 0.999879 & 1.000 \\
\hline 0.004 & 0.999958 & 0.99971 & 0.999998 \\
\hline 0.005 & 0.999917 & 0.999432 & 0.999996 \\
\hline 0.006 & 0.999856 & 0.99902 & 0.999991 \\
\hline 0.007 & 0.99977 & 0.99845 & 0.999984 \\
\hline 0.008 & 0.999655 & 0.9977 & 0.999972 \\
\hline 0.009 & 0.999509 & 0.996749 & 0.999954 \\
\hline 0.01 & 0.999327 & 0.995579 & 0.99993 \\
\hline 0.02 & 0.995506 & 0.971104 & 0.999171 \\
\hline 0.03 & 0.985057 & 0.918064 & 0.995381 \\
\hline 0.04 & 0.966795 & 0.845963 & 0.985617 \\
\hline 0.05 & 0.940898 & 0.767694 & 0.967553 \\
\hline 0.06 & 0.908673 & 0.692451 & 0.940579 \\
\hline 0.07 & 0.871984 & 0.624608 & 0.905915 \\
\hline 0.08 & 0.832751 & 0.565343 & 0.865905 \\
\hline 0.09 & 0.792637 & 0.514266 & 0.823133 \\
\hline 0.1 & 0.75292 & 0.4704 & 0.779798 \\
\hline
\end{tabular}

Case 1: fix $\alpha=0.2 \lambda$ and $\mu=0.1$, vary the values of $\lambda \in\{0.001, \ldots, 0.1\}$ at $k=1,2,3$.
Case 2: fix $\alpha=0.2 \lambda$ and $\lambda=0.001$, vary the values of $\mu \in\{0.06, \ldots, 0.16\}$ at $k=1,2,3$.

Case 3: fix $\mu=0.1$ and $\lambda=0.001$, vary the values of $k \in\{1, \ldots, 8\}$.

Table 3. Comparison of $A v_{i}(\infty)$ by using three configurations when $\alpha=0.2 \lambda, \mu=0.1$ and $k=1$

\begin{tabular}{cccc}
\hline$\lambda$ & $A v_{1}(\infty)$ & $A v_{2}(\infty)$ & $A v_{3}(\infty)$ \\
\hline 0.001 & 0.999999 & 0.999991 & 1.000 \\
0.002 & 0.999991 & 0.999932 & 1.000 \\
0.003 & 0.999966 & 0.99977 & 0.999999 \\
0.004 & 0.999919 & 0.999459 & 0.999995 \\
0.005 & 0.999842 & 0.998959 & 0.999988 \\
0.006 & 0.999727 & 0.998235 & 0.999975 \\
0.007 & 0.999568 & 0.997255 & 0.999954 \\
0.008 & 0.999359 & 0.995994 & 0.999922 \\
0.009 & 0.999096 & 0.994429 & 0.999875 \\
0.01 & 0.998771 & 0.992545 & 0.999811 \\
0.02 & 0.992426 & 0.957671 & 0.998069 \\
0.03 & 0.976729 & 0.892932 & 0.990726 \\
0.04 & 0.951806 & 0.814595 & 0.974674 \\
0.05 & 0.919313 & 0.735678 & 0.948951 \\
0.06 & 0.881643 & 0.663011 & 0.91485 \\
0.07 & 0.841152 & 0.598937 & 0.874934 \\
0.08 & 0.799778 & 0.54351 & 0.831965 \\
0.09 & 0.758932 & 0.495871 & 0.78826 \\
0.1 & 0.719546 & 0.454912 & 0.745468 \\
\hline & & &
\end{tabular}

Table 4. Comparison of $A v_{i}(\infty)$ by using three configurations when $\alpha=0.2 \lambda, \mu=0.1$ and $k=2$ 
Table 5. Comparison of $A v_{i}(\infty)$ by using three configurations when $\alpha=0.2 \lambda, \mu=0.1$ and $k=3$

\begin{tabular}{cccc}
\hline$\lambda$ & $A v_{1}(\infty)$ & $A v_{2}(\infty)$ & $A v_{3}(\infty)$ \\
\hline 0.001 & 0.999999 & 0.999996 & 1.000 \\
0.002 & 0.999996 & 0.999974 & 1.000 \\
0.003 & 0.999987 & 0.999908 & 1.000 \\
0.004 & 0.999969 & 0.999779 & 0.999999 \\
0.005 & 0.999937 & 0.999565 & 0.999998 \\
0.006 & 0.999891 & 0.999245 & 0.999995 \\
0.007 & 0.999825 & 0.998797 & 0.99999 \\
0.008 & 0.999738 & 0.998204 & 0.999982 \\
0.009 & 0.999625 & 0.997445 & 0.999971 \\
0.01 & 0.999484 & 0.996503 & 0.999955 \\
0.02 & 0.996443 & 0.975751 & 0.999439 \\
0.03 & 0.987786 & 0.927628 & 0.996664 \\
0.04 & 0.972029 & 0.858568 & 0.988983 \\
0.05 & 0.948842 & 0.780833 & 0.973811 \\
0.06 & 0.91904 & 0.704505 & 0.949846 \\
0.07 & 0.884176 & 0.634948 & 0.917614 \\
0.08 & 0.846067 & 0.573927 & 0.879105 \\
0.09 & 0.806427 & 0.521301 & 0.836902 \\
0.1 & 0.766664 & 0.476157 & 0.793417 \\
\hline
\end{tabular}

Table 6. Comparison of $A v_{i}(\infty)$ by using three configurations when $\alpha=0.2 \lambda, \lambda=0.001$ and $k=1$

\begin{tabular}{cccc}
\hline$\mu$ & $A v_{1}(\infty)$ & $A v_{2}(\infty)$ & $A v_{3}(\infty)$ \\
\hline 0.06 & 0.999993 & 0.999957 & 1.000 \\
0.07 & 0.999996 & 0.999973 & 1.000 \\
0.08 & 0.999997 & 0.999982 & 1.000 \\
0.09 & 0.999998 & 0.999987 & 1.000 \\
0.1 & 0.999999 & 0.999991 & 1.000 \\
0.11 & 0.999999 & 0.999993 & 1.000 \\
0.12 & 0.999999 & 0.999995 & 1.000 \\
0.13 & 0.999999 & 0.999996 & 1.000 \\
0.14 & 0.999999 & 0.999997 & 1.000 \\
0.15 & 1.000 & 0.999997 & 1.000 \\
0.16 & 1.000 & 0.999999 & 1.000 \\
\hline
\end{tabular}

Table 7. Comparison of $A v_{i}(\infty)$ by using three configurations when $\alpha=0.2 \lambda, \lambda=0.001$ and $k=2$

\begin{tabular}{cccc}
\hline$\mu$ & $A v_{1}(\infty)$ & $A v_{2}(\infty)$ & $A v_{3}(\infty)$ \\
\hline 0.06 & 0.999997 & 0.999978 & 1.000 \\
0.07 & 0.999998 & 0.999986 & 1.000 \\
0.08 & 0.999999 & 0.999991 & 1.000 \\
0.09 & 0.999999 & 0.999993 & 1.000 \\
0.1 & 0.999999 & 0.999995 & 1.000 \\
0.11 & 0.999999 & 0.999996 & 1.000 \\
0.12 & 1.000 & 0.999997 & 1.000 \\
0.13 & 1.000 & 0.999998 & 1.000 \\
0.14 & 1.000 & 0.999998 & 1.000 \\
0.15 & 1.000 & 0.999999 & 1.000 \\
0.16 & 1.000 & 0.999999 & 1.000 \\
\hline
\end{tabular}

Table 8. Comparison of $A v_{i}(\infty)$ by using three configurations when $\alpha=0.2 \lambda, \lambda=0.001$ and $k=3$

\begin{tabular}{cccc}
\hline$\mu$ & $A v_{1}(\infty)$ & $A v_{2}(\infty)$ & $A v_{3}(\infty)$ \\
\hline 0.06 & 0.999998 & 0.999983 & 1.000 \\
0.07 & 0.999998 & 0.99999 & 1.000 \\
0.08 & 0.999999 & 0.999993 & 1.000 \\
0.09 & 0.999999 & 0.999995 & 1.000 \\
0.1 & 0.999999 & 0.999996 & 1.000 \\
0.11 & 1.000 & 0.999997 & 1.000 \\
0.12 & 1.000 & 0.999998 & 1.000 \\
0.13 & 1.000 & 0.999998 & 1.000 \\
0.14 & 1.000 & 0.999999 & 1.000 \\
0.15 & 1.000 & 0.999999 & 1.000 \\
0.16 & 1.000 & 0.999999 & 1.000 \\
\hline
\end{tabular}

Table 9. Comparison of configurations 1,2,3, for $A v_{i}(\infty)$

\begin{tabular}{cc|}
\hline \multicolumn{2}{|c|}{ Result } \\
$\lambda \in\{0.001, \ldots, 0.1\}, k \in\{1,2,3\}$ & $A v_{3}(\infty)>A v_{1}(\infty)>A v_{2}(\infty)$ \\
$\mu \in\{0.06, \ldots, 0.14\}$, at $k=1$ & $A v_{3}(\infty)>A v_{1}(\infty)>A v_{2}(\infty)$ \\
$\mu \in\{0.15,0.16\}$, at $k=1$ & $A v_{3}(\infty)=A v_{1}(\infty)>A v_{2}(\infty)$ \\
$\mu \in\{0.06, \ldots, 0.11\}$, at $k=2$ & $A v_{3}(\infty)>A v_{1}(\infty)>A v_{2}(\infty)$ \\
$\mu \in\{0.12, \ldots, 0.16\}$, at $k=2$ & $A v_{3}(\infty)=A v_{1}(\infty)>A v_{2}(\infty)$ \\
$\mu \in\{0.06, \ldots, 0.1\}$, at $k=3$ & $A v_{3}(\infty)>A v_{1}(\infty)>A v_{2}(\infty)$ \\
$\mu \in\{0.11, \ldots, 0.16\}$, at $k=3$ & $A v_{3}(\infty)=A v_{1}(\infty)>A v_{2}(\infty)$ \\
$k \in\{1,2,3\}$ & $A v_{3}(\infty)>A v_{1}(\infty)>A v_{2}(\infty)$ \\
$k \in\{4, \ldots, 8\}$ & $A v_{3}(\infty)=A v_{1}(\infty)>A v_{2}(\infty)$ \\
\hline
\end{tabular}

\section{Cost/ benefit ratio comparisons}

Let $b_{i}=C_{i} / A v_{i}(\infty)$, where $C_{i}$ be the cost of configuration $i$ for $i=1,2,3$ which are listed in Table 2 . We compare $b_{i}$, $i=1,2,3$, in three cases as follow:

Table 10. Comparison of $b_{i}$ by using three configurations when $\alpha=0.2 \lambda, \mu=0.1$ and $k=1$

\begin{tabular}{cccc}
\hline$\lambda$ & $b_{1}$ & $b_{2}$ & $b_{3}$ \\
\hline 0.001 & $2.0 \times 10^{7}$ & $1.50001 \times 10^{7}$ & $2.4 \times 10^{7}$ \\
0.002 & $2.00002 \times 10^{7}$ & $1.50001 \times 10^{7}$ & $2.4 \times 10^{7}$ \\
0.003 & $2.00007 \times 10^{7}$ & $1.50035 \times 10^{7}$ & $2.4 \times 10^{7}$ \\
0.004 & $2.00016 \times 10^{7}$ & $1.50081 \times 10^{7}$ & $2.40001 \times 10^{7}$ \\
0.005 & $2.00032 \times 10^{7}$ & $1.50156 \times 10^{7}$ & $2.40003 \times 10^{7}$ \\
0.006 & $2.00055 \times 10^{7}$ & $1.50265 \times 10^{7}$ & $2.40006 \times 10^{7}$ \\
0.007 & $2.00086 \times 10^{7}$ & $1.50413 \times 10^{7}$ & $2.40011 \times 10^{7}$ \\
0.008 & $2.00128 \times 10^{7}$ & $1.50603 \times 10^{7}$ & $2.40019 \times 10^{7}$ \\
0.009 & $2.00181 \times 10^{7}$ & $1.5084 \times 10^{7}$ & $2.4003 \times 10^{7}$ \\
0.01 & $2.00246 \times 10^{7}$ & $1.51127 \times 10^{7}$ & $2.40045 \times 10^{7}$ \\
0.02 & $2.01526 \times 10^{7}$ & $1.5663 \times 10^{7}$ & $2.40464 \times 10^{7}$ \\
0.03 & $2.04765 \times 10^{7}$ & $1.67986 \times 10^{7}$ & $2.42247 \times 10^{7}$ \\
0.04 & $2.10127 \times 10^{7}$ & $1.84141 \times 10^{7}$ & $2.46236 \times 10^{7}$ \\
0.05 & $2.17554 \times 10^{7}$ & $2.03894 \times 10^{7}$ & $2.52911 \times 10^{7}$ \\
0.06 & $2.26849 \times 10^{7}$ & $2.26241 \times 10^{7}$ & $2.62338 \times 10^{7}$ \\
0.07 & $2.37769 \times 10^{7}$ & $2.50444 \times 10^{7}$ & $2.74307 \times 10^{7}$ \\
0.08 & $2.50069 \times 10^{7}$ & $2.75984 \times 10^{7}$ & $2.88474 \times 10^{7}$ \\
0.09 & $2.63528 \times 10^{7}$ & $3.02498 \times 10^{7}$ & $3.04468 \times 10^{7}$ \\
0.1 & $2.77953 \times 10^{7}$ & $3.29734 \times 10^{7}$ & $3.21946 \times 10^{7}$ \\
\hline
\end{tabular}

Table 11. Comparison of $b_{i}$ by using three configurations when $\alpha=0.2 \lambda, \mu=0.1$ and $k=2$

\begin{tabular}{cccc}
\hline$\lambda$ & $b_{1}$ & $b_{2}$ & $b_{3}$ \\
\hline 0.001 & $2.0 \times 10^{7}$ & $1.50001 \times 10^{7}$ & $2.4 \times 10^{7}$ \\
0.002 & $2.00001 \times 10^{7}$ & $1.50005 \times 10^{7}$ & $2.4 \times 10^{7}$ \\
0.003 & $2.00003 \times 10^{7}$ & $1.50018 \times 10^{7}$ & $2.4 \times 10^{7}$ \\
0.004 & $2.00008 \times 10^{7}$ & $1.50044 \times 10^{7}$ & $2.4 \times 10^{7}$ \\
0.005 & $2.00017 \times 10^{7}$ & $1.50085 \times 10^{7}$ & $2.40001 \times 10^{7}$ \\
0.006 & $2.00029 \times 10^{7}$ & $1.50147 \times 10^{7}$ & $2.40002 \times 10^{7}$ \\
0.007 & $2.00046 \times 10^{7}$ & $1.50233 \times 10^{7}$ & $2.40004 \times 10^{7}$ \\
0.008 & $2.00069 \times 10^{7}$ & $1.50346 \times 10^{7}$ & $2.40007 \times 10^{7}$ \\
0.009 & $2.00098 \times 10^{7}$ & $1.50489 \times 10^{7}$ & $2.40011 \times 10^{7}$ \\
0.01 & $2.00135 \times 10^{7}$ & $1.50666 \times 10^{7}$ & $2.40017 \times 10^{7}$ \\
0.02 & $2.00903 \times 10^{7}$ & $1.54463 \times 10^{7}$ & $2.40199 \times 10^{7}$ \\
0.03 & $2.03034 \times 10^{7}$ & $1.63387 \times 10^{7}$ & $2.41114 \times 10^{7}$ \\
0.04 & $2.06869 \times 10^{7}$ & $1.77313 \times 10^{7}$ & $2.43502 \times 10^{7}$ \\
0.05 & $2.12563 \times 10^{7}$ & $1.9539 \times 10^{7}$ & $2.48048 \times 10^{7}$ \\
0.06 & $2.20101 \times 10^{7}$ & $2.16622 \times 10^{7}$ & $2.55162 \times 10^{7}$ \\
0.07 & $2.29362 \times 10^{7}$ & $2.40151 \times 10^{7}$ & $2.64926 \times 10^{7}$ \\
0.08 & $2.40168 \times 10^{7}$ & $2.65326 \times 10^{7}$ & $2.77167 \times 10^{7}$ \\
0.09 & $2.52322 \times 10^{7}$ & $2.91678 \times 10^{7}$ & $2.91569 \times 10^{7}$ \\
0.1 & $2.65633 \times 10^{7}$ & $3.18877 \times 10^{7}$ & $3.07772 \times 10^{7}$ \\
\hline
\end{tabular}


Table 12. Comparison of $b_{i}$ by using three configurations when $\alpha=0.2 \lambda, \mu=0.1$ and $k=3$.

\begin{tabular}{cccc}
\hline$\lambda$ & $b_{1}$ & $b_{2}$ & $b_{3}$ \\
\hline 0.001 & $2.0 \times 10^{7}$ & $1.50001 \times 10^{7}$ & $2.4 \times 10^{7}$ \\
0.002 & $2.00001 \times 10^{7}$ & $1.50004 \times 10^{7}$ & $2.4 \times 10^{7}$ \\
0.003 & $2.00003 \times 10^{7}$ & $1.50014 \times 10^{7}$ & $2.4 \times 10^{7}$ \\
0.004 & $2.00006 \times 10^{7}$ & $1.50033 \times 10^{7}$ & $2.4 \times 10^{7}$ \\
0.005 & $2.00013 \times 10^{7}$ & $1.50065 \times 10^{7}$ & $2.40001 \times 10^{7}$ \\
0.006 & $2.00022 \times 10^{7}$ & $1.50113 \times 10^{7}$ & $2.40001 \times 10^{7}$ \\
0.007 & $2.00035 \times 10^{7}$ & $1.50181 \times 10^{7}$ & $2.40002 \times 10^{7}$ \\
0.008 & $2.00053 \times 10^{7}$ & $1.5027 \times 10^{7}$ & $2.40004 \times 10^{7}$ \\
0.009 & $2.00075 \times 10^{7}$ & $1.50384 \times 10^{7}$ & $2.40007 \times 10^{7}$ \\
0.01 & $2.00103 \times 10^{7}$ & $1.50526 \times 10^{7}$ & $2.40011 \times 10^{7}$ \\
0.02 & $2.00714 \times 10^{7}$ & $1.53728 \times 10^{7}$ & $2.40135 \times 10^{7}$ \\
0.03 & $2.02473 \times 10^{7}$ & $1.61703 \times 10^{7}$ & $2.40803 \times 10^{7}$ \\
0.04 & $2.05755 \times 10^{7}$ & $1.74709 \times 10^{7}$ & $2.42674 \times 10^{7}$ \\
0.05 & $2.10783 \times 10^{7}$ & $1.92103 \times 10^{7}$ & $2.46454 \times 10^{7}$ \\
0.06 & $2.17618 \times 10^{7}$ & $2.12915 \times 10^{7}$ & $2.52673 \times 10^{7}$ \\
0.07 & $2.26199 \times 10^{7}$ & $2.3624 \times 10^{7}$ & $2.61548 \times 10^{7}$ \\
0.08 & $2.36388 \times 10^{7}$ & $2.61357 \times 10^{7}$ & $2.73005 \times 10^{7}$ \\
0.09 & $2.48008 \times 10^{7}$ & $2.87741 \times 10^{7}$ & $2.86772 \times 10^{7}$ \\
0.1 & $2.6087 \times 10^{7}$ & $3.15022 \times 10^{7}$ & $3.02489 \times 10^{7}$ \\
\hline & & & \\
\hline
\end{tabular}

Table 13. Comparison of $b_{i}$ by using three configurations when $\alpha=0.2 \lambda, \lambda=0.001$ and $k=1$

\begin{tabular}{cccc}
\hline$\mu$ & $b_{1}$ & $b_{2}$ & $b_{3}$ \\
\hline 0.06 & $2.00001 \times 10^{7}$ & $1.50006 \times 10^{7}$ & $2.4 \times 10^{7}$ \\
0.07 & $2.00001 \times 10^{7}$ & $1.50004 \times 10^{7}$ & $2.4 \times 10^{7}$ \\
0.08 & $2.00001 \times 10^{7}$ & $1.50003 \times 10^{7}$ & $2.4 \times 10^{7}$ \\
0.09 & $2.0 \times 10^{7}$ & $1.50002 \times 10^{7}$ & $2.4 \times 10^{7}$ \\
0.1 & $2.0 \times 10^{7}$ & $1.50001 \times 10^{7}$ & $2.4 \times 10^{7}$ \\
0.11 & $2.0 \times 10^{7}$ & $1.50001 \times 10^{7}$ & $2.4 \times 10^{7}$ \\
0.12 & $2.0 \times 10^{7}$ & $1.50001 \times 10^{7}$ & $2.4 \times 10^{7}$ \\
0.13 & $2.0 \times 10^{7}$ & $1.50001 \times 10^{7}$ & $2.4 \times 10^{7}$ \\
0.14 & $2.0 \times 10^{7}$ & $1.50001 \times 10^{7}$ & $2.4 \times 10^{7}$ \\
0.15 & $2.0 \times 10^{7}$ & $1.5 \times 10^{7}$ & $2.4 \times 10^{7}$ \\
0.16 & $2.0 \times 10^{7}$ & $1.5 \times 10^{7}$ & $2.4 \times 10^{7}$ \\
\hline
\end{tabular}

Table 14. Comparison of $b_{i}$ by using three configurations when $\alpha=0.2 \lambda, \lambda=0.001$ and $k=2$.

\begin{tabular}{cccc}
\hline$\mu$ & $b_{1}$ & $b_{2}$ & $b_{3}$ \\
\hline 0.06 & $2.00001 \times 10^{7}$ & $1.50003 \times 10^{7}$ & $2.4 \times 10^{7}$ \\
0.07 & $2.0 \times 10^{7}$ & $1.50002 \times 10^{7}$ & $2.4 \times 10^{7}$ \\
0.08 & $2.0 \times 10^{7}$ & $1.50001 \times 10^{7}$ & $2.4 \times 10^{7}$ \\
0.09 & $2.0 \times 10^{7}$ & $1.50001 \times 10^{7}$ & $2.4 \times 10^{7}$ \\
0.1 & $2.0 \times 10^{7}$ & $1.50001 \times 10^{7}$ & $2.4 \times 10^{7}$ \\
0.11 & $2.0 \times 10^{7}$ & $1.50001 \times 10^{7}$ & $2.4 \times 10^{7}$ \\
0.12 & $2.0 \times 10^{7}$ & $1.5 \times 10^{7}$ & $2.4 \times 10^{7}$ \\
0.13 & $2.0 \times 10^{7}$ & $1.5 \times 10^{7}$ & $2.4 \times 10^{7}$ \\
0.14 & $2.0 \times 10^{7}$ & $1.5 \times 10^{7}$ & $2.4 \times 10^{7}$ \\
0.15 & $2.0 \times 10^{7}$ & $1.5 \times 10^{7}$ & $2.4 \times 10^{7}$ \\
0.16 & $2.0 \times 10^{7}$ & $1.5 \times 10^{7}$ & $2.4 \times 10^{7}$ \\
\hline
\end{tabular}

Table 15. Comparison of $b_{i}$ by using three configurations when $\alpha=0.2 \lambda, \lambda=0.001$ and $k=3$.

\begin{tabular}{cccc}
\hline$\mu$ & $b_{1}$ & $b_{2}$ & $b_{3}$ \\
\hline 0.06 & $2.0 \times 10^{7}$ & $1.50002 \times 10^{7}$ & $2.4 \times 10^{7}$ \\
0.07 & $2.0 \times 10^{7}$ & $1.50002 \times 10^{7}$ & $2.4 \times 10^{7}$ \\
0.08 & $2.0 \times 10^{7}$ & $1.50001 \times 10^{7}$ & $2.4 \times 10^{7}$ \\
0.09 & $2.0 \times 10^{7}$ & $1.50001 \times 10^{7}$ & $2.4 \times 10^{7}$ \\
0.1 & $2.0 \times 10^{7}$ & $1.50001 \times 10^{7}$ & $2.4 \times 10^{7}$ \\
0.11 & $2.0 \times 10^{7}$ & $1.5 \times 10^{7}$ & $2.4 \times 10^{7}$ \\
0.12 & $2.0 \times 10^{7}$ & $1.5 \times 10^{7}$ & $2.4 \times 10^{7}$ \\
0.13 & $2.0 \times 10^{7}$ & $1.5 \times 10^{7}$ & $2.4 \times 10^{7}$ \\
0.14 & $2.0 \times 10^{7}$ & $1.5 \times 10^{7}$ & $2.4 \times 10^{7}$ \\
0.15 & $2.0 \times 10^{7}$ & $1.5 \times 10^{7}$ & $2.4 \times 10^{7}$ \\
0.16 & $2.0 \times 10^{7}$ & $1.5 \times 10^{7}$ & $2.4 \times 10^{7}$ \\
\hline
\end{tabular}

Numerical results of the $b_{i}$ for configuration $i(i=1,2,3)$ are calculated in Tables $10-15$ for three cases, respectively. Tables 10-12 show that the $b_{i}$ increases as $\lambda$ or $\alpha$ increases for any configuration. On the other hand, Tables 13-15 show that the $b_{i}$ decreases as $\mu$ increase for any configuration. One observes from Tables 10-12 that optimal configuration using $b_{i}$ value depends on the value of $\lambda$. When $\lambda \in\{0.001, \ldots, 0.06\}$ at $k \in\{1,2,3\}$, the optimal configuration is configuration 2 , but when $\lambda \in\{0.07, \ldots, 0.09,0.1\}$ at $k \in\{1,2,3\}$, the optimal configuration is configuration 1 . It appears from Tables 13-15 that the optimal configuration using the $b_{i}$ value does not depend on the value of $\mu$. We see from Tables 13-15 that the optimal configuration using the $b_{i}$ value is configuration 2 .

\section{Conclusions}

In this paper, we constructed three different series system configurations with mixed standby components and $k$-stage Erlang repair times to study the $b_{i}$ analysis of three configurations. We developed the explicit expressions for the steady-state availability, $A v_{i}(\infty)$, for three configurations and performed a comparative analysis for them. We rank three configurations based on the $A v_{i}(\infty)$, and the $b_{i}$. Using the $b_{i}$ measure, the optimal configuration depends on the values of $\lambda$ and $\alpha$ but does not depend on the value of $\mu$.

\section{REFERENCES}

[1] Chandrasekhar, P., Natarajan, R., and Yadavalli, V. S. S., 2004. A Study on a two unit standby system with Erlangian repair time. Asia-Pacific Journal of Operational Research, 21, 271-277.

[2] Goel, L. R., and Gupta, P., 1984. Stochastic analysis of a two-unit parallel system with partial and catastrophic failures and preventive maintenance. Microelectronics and Reliability. 24, 881-883.

[3] Goel, L. R., Sharma, G. C., and Gupta, P., 1985. Cost analysis of a two unit cold standby system under different weather conditions. Microelectronics and Reliability. 25, 655-659. 
[4] Gopalan, M. N. and Natesan, J., 1982. Cost-benefit analysis of a 1-server 2-unit system subject to different repair strategies. Microelectronics and Reliability, 22, 393-397.

[5] Khaled, M. El-Said. and El-Sherbeny, M. Salah., 2005. Evaluation of Reliability \& Availability Characteristics of two Different Systems by Using Linear First Order Differential Equations. Journal of Mathematics and Statistics. 1, 119-123.

[6] Khaled, M. EL-Said. and El-Sherbeny, M. Salah., 2006. Comparing of reliability characteristic between two different systems. Applied Mathematics and Computation. 173, 1183-1199.

[7] Kuo-Hsiung Wang, Ching-Chang Kuo., 2000. Cost and probabilistic analysis of series systems with mixed standby components. Applied Mathematical Modelling. 24, 957-967.
[8] Mokaddis, G. S., El-Sherbeny, M. Salah. and Y. Mahmoud Ayid., 2009. Stochastic behavior of a two-unit warm standby system with two types of repairmen and patience time. Journal of Mathematics and Statistics. 5, 42-46.

[9] Mokaddis, G. S., El-Sherbeny, M. Salah. and Entesar Al-Esayeh., 2009. Stochastic Behavior of Redundant Complex System with Two Types of Failure. Journal of Mathematics and Statistics. 5, 112-117.

[10] El-Sherbeny, M. Salah., 2010. The optimal system for series systems with mixed standby components. Journal of Quality in Maintenance Engineering. 16, 319- 334.

[11] Nianfu Yang, B. S. Dhillon, 1995. Availability analysis of a repairable standby human-machine system. Microelectronics and Reliability. 35, 1401-1413. 